Gut, 1978, 19, 132-136

\title{
Selective angiography in acute mid-gut ischaemia
}

\author{
D. R. HARPER ${ }^{1}$ AND T. A. S. BUIST \\ From the Royal Infirmary of Edinburgh, Edinburgh
}

SUMMARY Acute mesenteric ischaemia has a high mortality. Early diagnosis and treatment appropriate to the type and extent of vascular insufficiency are essential. Selective angiography can establish the diagnosis and distinguish patients with acute vascular occlusion who require urgent surgery from those with non-occlusive ischaemia for which early surgery is contraindicated.

Acute mesenteric vascular occlusion continues to carry an unacceptably high mortality (Mavor et al., 1962; Aakhus and Brabrand, 1967; Marston, 1971; Wittenberg, et al., 1973; Boley et al., 1973; Singh et al., 1975), this being largely because of the diagnostic problem it presents (Recek et al., 1968; Mavor et al., 1972). Clinical diagnostic pointers are well known (Marston, 1971; Mavor, 1972) but are non-specific. These include severe epigastric or periumbilical pain which may be constant or, less often, colicky in nature, and vomiting or diarrhoea, the latter unassociated with blood in the early stages. Perhaps most significant is the paucity of abdominal signs in contrast to the severity of symptoms in a patient who can be effectively treated. Circulatory failure, peritonism, leucocytosis, and acidosis (Marston, 1971; Williams, 1971; Jamieson et al., 1975) identify the patients with advanced irreversible ischaemia.

Three pathological types of acute arterial insufficiency are recognised-namely, acute embolism, acute thrombosis, and non-occlusive ischaemia. Acute embolism occurs in association with cardiovascular disease elsewhere; the embolus is usually lodged at or beyond the origin of the middle colic branch. The proximal artery is regular, the contrast cut-off distinct, and collaterals are absent. Acute thrombosis is more frequently the end stage of origin stenosis (Mavor, 1959) and prodromal symptoms are frequent. The severity of the final insult will relate to the imbalance between rapidity of occlusion to the development of adequate collaterals. Usually, even in acute decompensation some evidence of a collateral round the head of the pancreas may be discerned, and there may be extensive visceral and limb atherosclerosis in

${ }^{1}$ Address for reprint requests: as above.

Received for publication 2 September 1977 addition. However, as with acute vascular occlusion in the lower limb, differentiation between embolism and acute thrombosis in the elderly patient may not be straightforward, even with adequate angiography. In the last decade, nonocclusive ischaemia has been described with increasing frequency and is usually associated with reduction in cardiac output from whatever cause, and digoxin or vasopressin therapy (Ende, 1958). Peripheral vasocontriction may also be associated with superior mesenteric embolism and may be evident in other visceral branches as well. The incidence of these lesions differs greatly in various series; Williams in 1971 suggested an incidence of up to $50 \%$ for non-occlusive cases, while four years later Singh et al. (1975) found thrombosis in twothirds and did not mention non-occlusion. These authors and others all agree, however, on a mortality figure of around $80 \%$ with treatment and virtually $100 \%$ without.

An energetic approach to the problem has been advocated for some time (Mavor, 1972) but only recently has a substantial improvement in mortality -and that only in the embolic group-been noted (Bergen et al., 1975). The expeditious management of acute mesenteric vascular occlusion would seem to depend on earlier precise diagnosis followed by a determined effort to revascularise rather than simply to ablate the affected small bowel. Earlier diagnosis will still depend on clinical awareness above all else. Precipitate laparotomy in the critically ill patient is as unacceptable as diagnostic vacillation. Arterial catheterisation may not only produce a precise angiographic diagnosis, but can also provide a route for therapy (Aakhus and Braband, 1967; Recek et al., 1968; Boley et al., 1973; Wittenberg et al., 1973; Bergan et al., 1975), although the value of the latter is not yet clearly established. We should like to report three cases in which early 132 
angiography played a decisive role in the diagnosis and management.

\section{Case 1}

Mrs, H., aged 61 years, was known to have mitral stenosis and atrial fibrillation. She experienced a sudden onset of severe epigastric pain and was seen in hospital $1 \frac{1}{2}$ hours later. A diagnosis of acute mesenteric vascular occlusion seemed likely but the diagnosis was confused by the prior administration of analgesic drugs. The plain film of abdomen showed an almost gas-free intestine-an appearance which is not uncommon in the early stages of acute mesenteric ischaemia. The patient was then referred for immediate angiography. An abdominal aortogram was followed by selective superior mesenteric arteriography and these demonstrated an embolus in the superior mesenteric artery producing complete occlusion of this vessel distal to the origin of the ileocolic artery. Incomplete embolic occlusions were also seen in jejunal branches, the right colic and ileocolic arteries (Fig. 1). No collateral circulation was demonstrated and there was clearly a

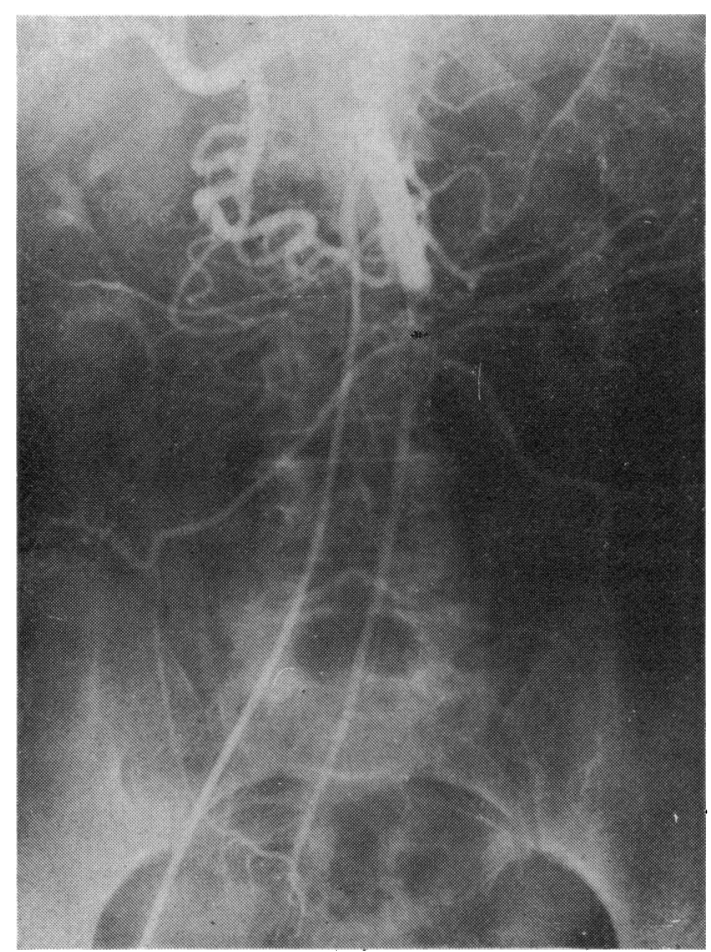

Fig. 1 Case 1. Selective superior mesenteric arteriogram. Embolus occludes the superior mesentric artery and extends into the origin of several small intestinal branches. severe degree of mesenteric ischaemia. The arteriogram also demonstrated occlusion of the coeliac artery at its origin with well-established collateral supply to the coelic circulation from pancreaticoduodenal branches of the superior mesenteric artery. The patient was then transferred to theatre. At laparotomy, the mid-gut loop was slightly blue but viable, the distal superior mesenteric pulse being absent. Embolic material and consecutive thrombus was removed through a proximal transverse arteriotomy and good back and forward flow established. The arteriotomy was closed and bowel perfusion became normal. The postoperative course was uneventful and the patient left hospital three weeks later. She remains well.

\section{Case 2}

Mr. R.B., aged 66 years, was referred to hospital with a 24-hour history of central abdominal pain and haematemesis. Examination revealed an agitated, confused, but normotensive man. He had a grade II systolic ejection murmur. His epigastrium was slightly tender and there was fresh blood in the rectum. A plain radiograph of the abdomen showed a normal large bowel pattern but two gas-filled

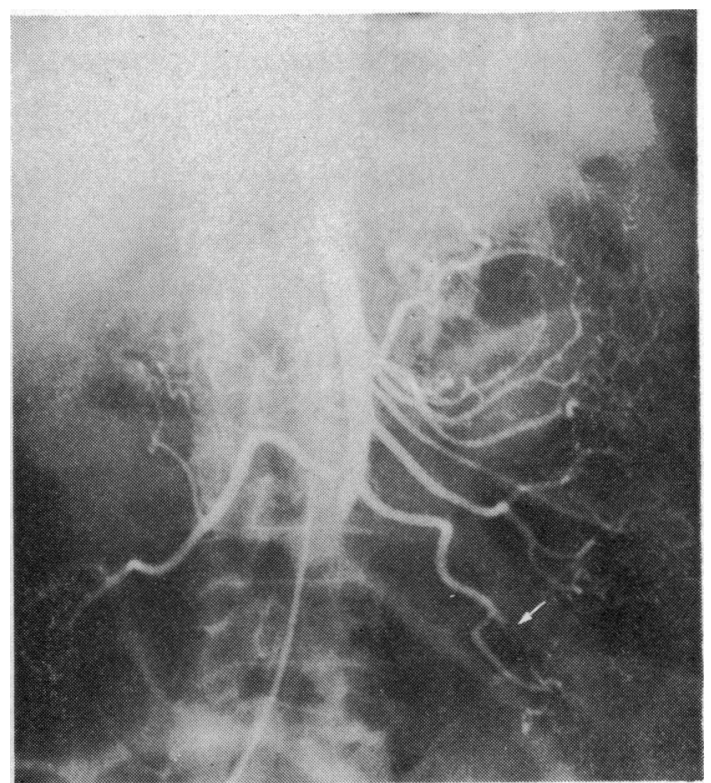

Fig. 2 Case 2. Selective superior mesenteric arteriogram. There is complete occlusion of the superior mesenteric artery distal to the ileocolic branch. A smaller embolic occlusion is shown within an ileal branch (arrow). 
loops of ileum were suggestive of local ileus with some thickening of the bowel wall. Acute mesenteric ischaemia was considered likely and the patient was referred for angiography. An abdominal aortogram was followed by selective superior and inferior mesenteric arteriography. The arteriograms demonstrated complete occlusion of the superior mesenteric artery distal to the ileocolic branch. Embolic occlusion was also seen in an upper ileal branch. There was no evidence of collateral circulation and the films indicated that there was a severe degree of small bowel ischaemia (Fig. 2). Laparotomy revealed an extensive length of small bowel which was ischaemic but viable, and the colon contained blood. The embolus, which could be palpated in the superior mesenteric artery, was removed along with associated thrombus via an infraduodenal arteriotomy. After closure of the vessel good flow was noted and the bowel colour improved dramatically. Twelve hours after surgery heparin infusion was started and the patient was digitalised at the onset of rapid atrial fibrillation. He gradually improved until the fourth day when he suddenly became shocked, hemiparetic, and anuric. He died on the fifth postoperative day. At necropsy death was found to be due to a large cerebral embolus.

\section{Case 3}

Mr. C.S., aged 56 years, was admitted to the Coronary Care Unit, with a diagnosis of acute myocardial infarction. Forty-eight hours later he experienced a sudden onset of severe, constant, periumbilical pain. There was no evidence of peritonism and the serum amylase was normal. A clinical diagnosis of acute mesenteric ischaemia was made, and the patient was referred for immediate angiography while arrangements for laparotomy were put in hand. Plain films of the abdomen were considered to be normal. Abdominal aortography was followed by selective superior mesenteric and coeliac arteriography. The superior mesenteric artery and its branches appeared to be generally attenuated with impaired filling of the peripheral intestinal branches, but there was no evidence of emboli or occlusion. These appearances were considered to be due to reactive vasospasm. The coeliac arteriogram showed emboli within right and left hepatic arteries producing only partial obstruction within these vessels (Fig. 3). Surgery was therefore not indicated. In the next few days the patient developed some right upper quadrant tenderness and his liver function tests suggested

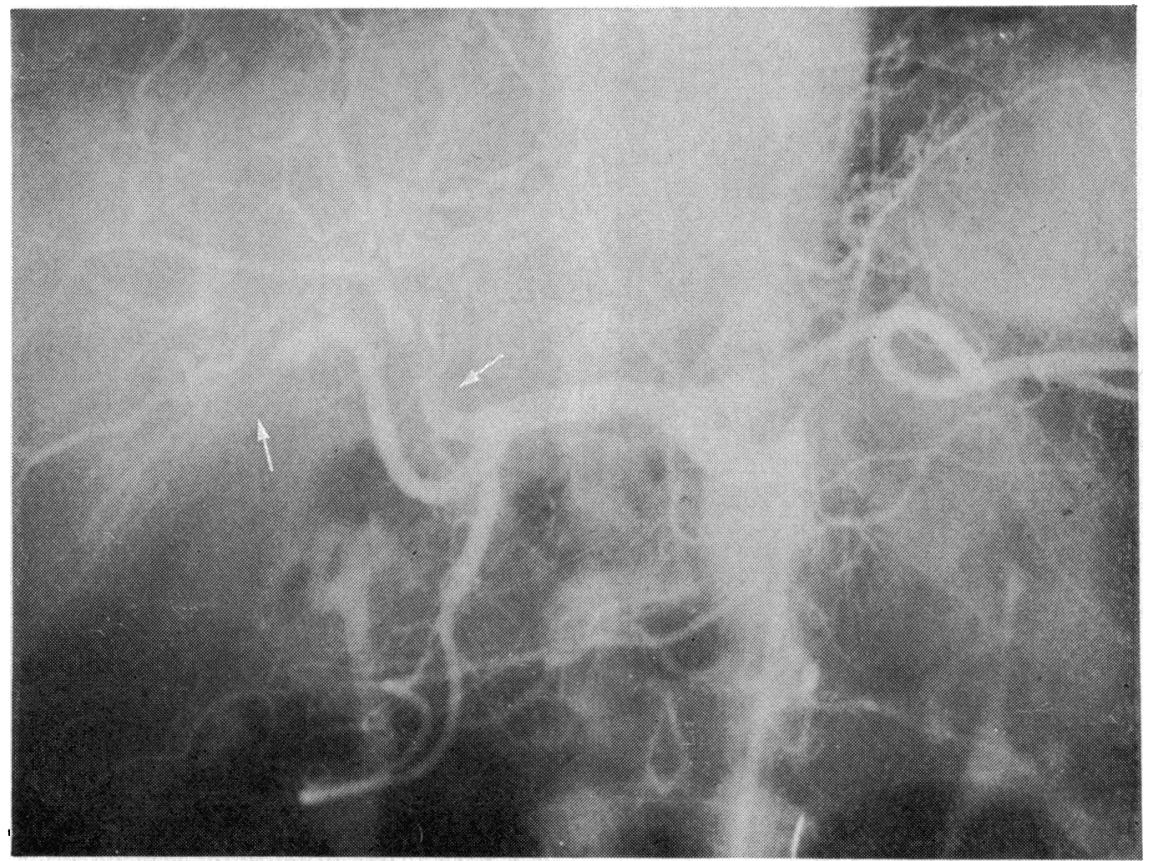

Fig. 3 Case 3. Selective coeliac arteriogram. Incompletely occluding emboli are seen within right and left hepatic arteries (arrows). 
hepatocellular damage. Both the clinical signs and serology returned to normal subsequently and he was transferred from the Coronary Care Unit. A further fatal myocardial infarct occurred the following day.

\section{Discussion}

Selective angiography provided early confirmatory diagnosis in all three patients. In the two requiring surgery, successful revascularisation obviated bowel resection. In neither case was a 'second look' operation deemed necessary-a decision which should be made at the initial exploration (Boley et al., 1973), and which should in any case be performed well within 24 hours (Williams, 1971). In the first case early diagnosis and treatment led to survival, reflecting Bergan's satisfactory results for an early group where embolectomy without bowel resection was rewarded with a survival of $73 \%$. The second case had an embolectomy only at 30 hours, successful revascularisation being achieved although the ischaemia was more advanced. This patient succumbed from a cerebral embolus at four days. Where bowel ischaemia has advanced significantly to warrant resection in spite of revascularisation, the mortality is inevitably higher and in Bergan's series $82 \%$. Non-operative survival is reported, the initial diagnosis being made clinically by Serjeant (1965), angiographically by Aakhus (1966), and at laparotomy by Daniel et al. (1966). These were all single case reports but that of Daniel is particularly interesting. Unequivocal evidence of bowel ischaemia was found at initial exploration and a regime of dextran 40 instituted. Further laparotomy at 48 hours showed a normal bowel perfusion. In the case reported by Aakhus angiography showed embolism to be multiple, minor, and peripheral and a possible alternative diagnosis of cholecystitis was entertained. It appears that main-stem embolic occlusion of a previously normal superior mesenteric artery is incompatible with survival without surgery.

The value of immediate angiography is not merely to confirm mesenteric vascular insufficiency but to define its nature. In non-occlusive ischaemia, early operation may be contraindicated (Williams, 1971), the emphasis being to restore cardiac output. Embolic occlusion requires prompt embolectomy almost invariably, while acute thrombosis merits planned revascularisation-usually by a vascular specialist. Recent interest has been focused on the patients with non-occlusive ischaemia, for here selective angiography may have a particular role to play by providing a route for the infusion of vasoactive substances and for angiographic review (Aakhus and Braband, 1967, Boley et al., 1973).
Pharmacoangiography may be relevant in occlusive as well as non-occlusive disease. Arterial constriction, in association with embolism, may well be controlled or improved by the infusion of papaverine or other vasodilators (Aakhus and Braband, 1967; Boley et al., 1973; Dencker et al., 1975) and the maintenance of the selective catheter position with a similar infusion after intraluminal manipulation at embolectomy has been recommended (Boley et al., 1973) and deserves further evaluation.

\section{Conclusion}

Acute mesenteric vascular insufficiency continues to be a challenging surgical emergency. The associated high mortality may be reduced not only by earlier clinical diagnosis but by the recognition of the nature of the lesion producing the ischaemia. Prompt angiography will define the lesion and its extent with precision, allowing a rational approach to individual cases in a way similar to its established role in peripheral vascular insufficiency.

The authors would like to thank Dr Irene Prossor for her contribution to the management of case 2 and Mr. I. B. Macleod and Mr. A. J. Duff for permission to include cases under their care.

\section{References}

Aakhus, T. (1966). The value of angiography in superior mesenteric artery embolism. British Journal of Radiology, 39, 928-932.

Aakhus, T., and Brabrand, G. (1967). Angiography in acute superior mesenteric arterial insufficiency. Acta Radiologica, (Diagnosis), 6, 1-12.

Bergan, J. J., Dean, R. H., Conn, J., Jr., and Yao, J. S. T. (1975) Revascularisation in the treatment of mesenteric infarction Annals of Surgery, 182, 430-438.

Boley, S. J., Sprayregan, S., Veith, F. J., and Siegelman, S. S. (1973). An aggressive roentgenologic and surgical approach to acute mesenteric ischemia. In Surgery Annual, vol. 5, pp. 355-378. Edited by L. M. Nyhus. Appleton-CenturyCrofts: New York.

Daniel, W. J., Mohamed, S. D., and Matheson, N. A. (1966). Treatment of mesenteric embolism with dextran 40 . Lancet, 1, 567-569.

Dencker, H., Göthlin, J., Hedner, P., Lunderquist, A., Norryd, C., and Tylen, U. (1975). Superior mesenteric angiography and blood flow following intra-arterial injection of prostaglandin $\mathrm{F}_{2}$. American Journal of Roentgenology, 125, 111-118.

Ende, N. (1958). Infarction of the bowel in cardiac failure. New England Journal of Medicine, 258, 879-881.

Jamieson, W. G., Lozon, A., Durand, D., and Wall, W. (1975). Changes in serum phosphate levels associated with intestinal infarction and necrosis. Surgery, Gynecology and Obstetrics, 140, 19-21.

Marston, A. (1971) Experimental aspects of acute superior mesenteric artery occlusion, In Vascular Disorders of the Intestine, pp. 345-357. Edited by S. J. Boley, S. S. Schwartz, and L. F. Williams, Jr. Butterworths: London.

Mavor, G. E. (1959). Stenosis of the superior mesenteric artery. Postgraduate Medical Journal, 35, 558-563. 
Mavor, G. E (1972). Acute occlusion of the superior mesenteric artery Clinics in Gastroenterology, 1, 639-653.

Mavor, G. E., Lyall, A. D., Chrystal, K. M. R., and Tsapogas M. (1962). Mesenteric infarction as a vascular emergency. The clinical problems. British Journal of Surgery, 50, 219-225.

Recek, C., Kren, V., Fixa, B., and Steinhart, L. (1968). Selective mesentericography as a guide to diagnosis and treatment of acute superior mesenteric artery occlusion. Journal of Cardiovascular Surgery (Torino), 9, 184-189.
Serjeant, J. C. B. (1965). Mesenteric embolus treated with low-molecular-weight dextran. Lancet, 1, 139-140.

Singh, R. P., Shah, R. C., Lee, S. T. (1975). Acute mesenteric vascular occlusion: a review of thirty-two patients. Surgery, 78, 613-617.

Williams, L. F. Jr. (1971). Vascular insufficiency of the intestines. Gastroenterology, 61, 757-777.

Wittenberg, J., Athanasoulis, C. A., Shapiro, J. H., and Williams, L. F., Jr. (1973). A radiological approach to the patient with acute extensive bowel ischemia. Radiologv, 106, 13-24. 